\title{
Late influences on perceptual grouping: Illusory figures
}

\author{
STEPHEN E. PALMER and ROLF NELSON \\ University of California, Berkeley, California
}

\begin{abstract}
Two experiments demonstrate that grouping can be strongly influenced by the presence of figures defined by illusory contours. Rectangular arrays were constructed in which a central column of figures could group either with those on one side, on the basis of perception of figures defined by illusory contours, or with those on the other side, on the basis of physically present inducing elements. In all displays, subjects grouped according to the illusory figures significantly more often than for control displays that contained the same inducing elements, but rearranged so that illusory contours were degraded or eliminated. A second experiment showed that in objectively defined grouping tasks, subjects grouped faster by illusory figures than by inducing elements. These results indicate that grouping can occur after illusory contours have been perceived.
\end{abstract}

In 1923, Max Wertheimer called attention to one of the fundamental problems of visual perception: How does the visual system organize the complex array of light striking the retina into the coherent spatial array of objects that we experience when we view the world? Although he did not by any means solve this complex and difficult problem, which persists to this day, he did make substantial inroads by identifying a number of stimulus-driven principles of grouping. These are the well-known "laws" of perceptual grouping (e.g., proximity, similarity, good continuation, and common fate) that are discussed in virtually all introductory textbooks. Other principles of grouping have since been added to this list, including common region (Palmer, 1992), element connectedness (Palmer \& Rock, 1994b), and synchrony (Palmer \& Levitin, 2000), but Wertheimer's original article has been the preeminent statement of both the problem and the nature of its solution for most of this century.

Recently, there has been interest in understanding how the mechanisms responsible for grouping fit within modern information processing theories of vision. Central to this task has been the attempt to specify where grouping operates along the temporal flow of visual processing (e.g., Palmer, 1999; Palmer \& Rock, 1994a, 1994b). Following Wertheimer's discussion, the standard assumption appears to have been that grouping processes operate fairly early in visual processing, within what would now be called a 2-D, image-based representation. For example, Marr's (1982) influential theory of vision placed at least

The preparation of this article was supported by Grant 1-R01MH46141 from the National Institute of Mental Health to S.E.P. We thank Steven Yantis, Thomas Sanocki, and two anonymous reviewers for their helpful comments on an earlier draft of this article. Correspondence should be addressed to S. E. Palmer, Psychology Department, University of California, Berkeley, CA 94720-1650 (e-mail: palmer@cogsci.berkeley.edu). some grouping operations right after the "raw primal sketch." He proposed that grouping processes organized the edges, lines, blobs, and terminators of the raw primal sketch into the more global elements of the "full primal sketch." Because both primal sketches are 2-D imagebased representations that precede construction of higher level surface-based and/or object-based representations, grouping operates before the perception of depth and occlusion relations in Marr's theory. Other theorists who have addressed the issue of when grouping occurs also place it early, typically proposing that it occurs before the operation of selective attention (e.g., Kahneman \& Henik, 1981; Neisser, 1967; Treisman, 1986). The rationale for this claim is that attention must select among a set of unified objects or elements and that grouping is needed to arrive at such objects or elements.

Palmer and Rock (1994a, 1994b) challenged the "earlyonly" view that all grouping occurs at an early (i.e., 2-D, preconstancy) stage of processing. First, they cited Rock and Brosgole's (1964) demonstration that grouping can be strongly influenced by binocularly perceived depth. In a dark room, subjects were shown flat rectangular arrays of luminous beads that were closer together vertically than horizontally when they were presented in the frontal plane. Under these conditions, all subjects saw them grouped vertically rather than horizontally because of proximity. Then subjects saw the same arrays slanted in depth (i.e., rotated around a vertical axis) so that horizontal distances were foreshortened. Subjects still reported seeing vertical columns of beads, even when their retinal projections were closer together horizontally than vertically. This result occurred only when subjects saw the displays with both eyes open, however, so that they perceived the array in stereoscopic depth. With only one eye open, subjects saw the beads group horizontally at precisely the slant at which the projected distances were smaller horizontally than vertically. Rock and Brosgole 
therefore concluded that the final perception of grouping must take place after depth perception based on binocular disparity.

A second telling result came from an experiment by Rock, Nijhawan, Palmer, and Tudor (1992), who addressed the analogous issue in the domain of achromatic color perception. They asked whether the important factor in grouping by color similarity is the retinally measured $l u$ minances of elements or their phenomenally perceived lightnesses after lightness constancy has been achieved. As illustrated in Figure 1, they used a cast shadow to decouple image luminance and perceived lightness. The critical display was carefully constructed so that the squares in the central column of the array were identical in physical reflectance to the squares on one side (i.e., they were made of paper that was the same shade of gray), but were seen under a shadow cast by an opaque vertical strip hanging nearby, so that their retinal luminance was identical to that of the darker squares on the other side. The results showed that grouping followed the predictions of the late (postconstancy) grouping hypothesis: Similarity grouping was governed by the perceived lightnesses of the squares rather than by their retinal luminances. The same result was obtained when the central squares were displayed behind a translucent strip of plastic instead of in a cast shadow. Control conditions effectively ruled out an alternative explanation that retinal luminance ratios were responsible for the obtained results.

More recently, Palmer, Neff, and Beck (1996) showed that grouping by shape similarity is affected by occlusion relations and visual completion phenomena. Using

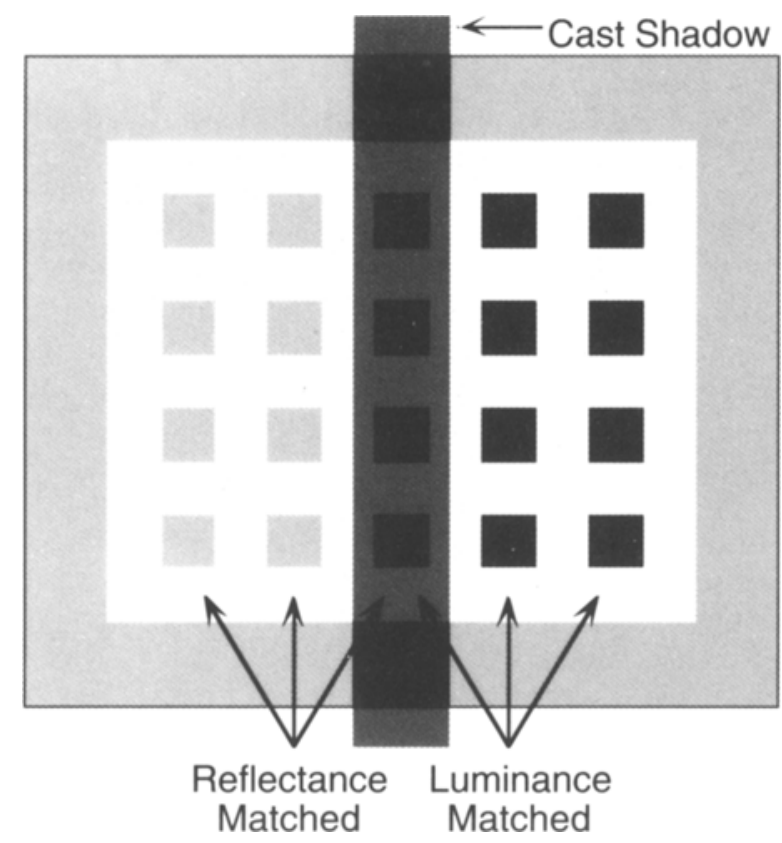

Figure 1. The critical display in an experiment on grouping and lightness constancy by Rock, Nijhawan, Palmer, and Tudor (1992). Subjects report seeing the middle column group to the left with reflectance-matched figures rather than to the right with luminance-matched regions. (See text for further details.) the same type of displays as Rock et al. (1992), these investigators constructed displays in which half-circles in the central column are generally perceived as whole circles partly occluded by a vertical strip, as shown in Figure 2 . An early view of grouping predicts that the central elements will still be seen to group with the half-circles on the left because both have the retinal shape of a halfcircle. A late view of grouping predicts that they will group with the full circles on the right because they are perceived as completed behind the occluding strip. The results showed a robust tendency for the central column of occluded figures to group with the full circles, indicating that grouping is strongly influenced by similarity of completed shape rather than retinal shape.

The purpose of the present study is to extend this line of inquiry about the locus of grouping by determining whether it is completed before or after the perception of illusory figures, such as the well-known Kanisza triangle. Although the explanation of illusory figures-that is, figures defined at least in part by zero-contrast contours rather than real ones - is controversial, one possibility is that they arise when the visual system encounters evidence that one surface occludes another in places where there is no contrast in luminance between the occluding surface and the occluded one behind it (Rock \& Anson, 1979). In the case of the Kanisza triangle, people perceive the edges of a white triangle not only where it partly occludes the three black inducing circles (via high-contrast edges), but also where it occludes the white background between the black circles (via zero-contrast edges). By this account, the perception of illusory figures must arise at a level of the visual system after the spatial integration of depth edges defined by occlusion. (The interested reader is referred to Lesher, 1995, and Spillmann \& Dresp, 1995, for reviews of the extensive literature on illusory contours.)

\section{EXPERIMENT 1 Subjective Grouping Reports}

The initial study employed the same type of subjective grouping paradigm as Rock et al. (1992) and Palmer et al. (1996). In this case, the central column of the array should be grouped with the elements on one side if grouping is based on the perception of illusory contours and figures, and on the other side if it is based on the perception of the contours of the inducing elements. Figure 3 illustrates the experimental stimuli. In each case, the central column of the display can be grouped either to the left (with similar shapes defined by illusory contours) or to the right (with similar shapes defined by the physical contours of the inducing elements). Two of the stimuli contain edge-induced illusory contours (Figures $3 \mathrm{~A}$ and $3 \mathrm{~B}$ ) and the other two contain line-induced illusory contours (Figures $3 \mathrm{C}$ and $3 \mathrm{D}$ ).

\section{Method \\ Subjects. Twenty-eight undergraduates from the University of California, Berkeley participated in the study. These students re-}




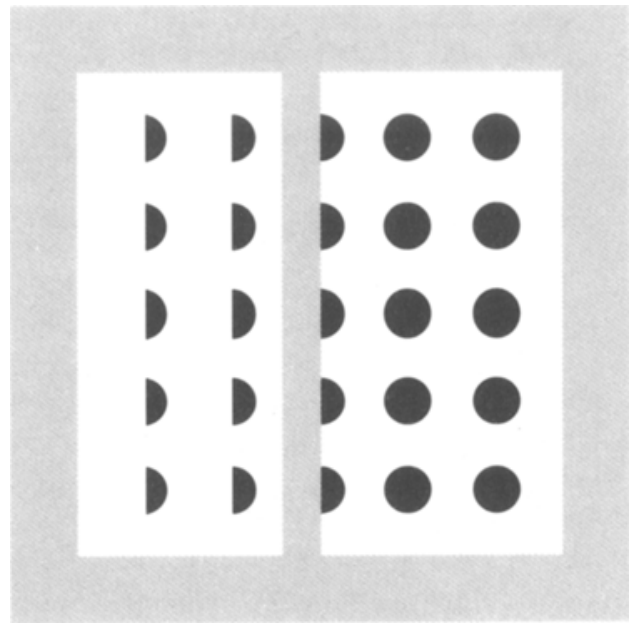

Figure 2. The critical display in an experiment on grouping and amodal completion by Palmer, Neff, and Beck (1996). Subjects report seeing the partly occluded middle column of retinal halfcircles group to the right with the retinally complete full circles rather than to the left with the retinally complete half-circles. (See text for further details.)

ceived course credit for one of several psychology courses. All were naive with respect to the experimental hypothesis, and all had normal or corrected-to-normal vision.

Materials. Stimuli were displayed on a 17-in. AppleVision monitor via a Macintosh Power PC 7500. Subjects sat approximately $2 \mathrm{ft}$ from the monitor. Stimuli were displayed via PsyScope, a presentation programming language (Cohen, MacWhinney, Flatt, \& Provost, 1993). Subjects first indicated their choice of grouping by pressing one of two buttons on a button box. They then rated the strength of this grouping by entering a number $(1=$ weak; $3=$ strong) on the computer keyboard.

Stimuli and Procedure. The figures within each display were arranged in rectangular arrays. The subjects' primary task was always to decide whether the middle column of figures grouped to the left or to the right. The four experimental types of arrays are shown in Figure 3. Each figure within an array is constructed of physically defined inducing elements configured to produce clear perceptions of illusory figures. For example, in Figure 3A, the illusory figures are rectangles (analogous to Kanisza triangles) that arise from configurations of inducing elements consisting of notched background ovals. The experimental arrays were constructed so that grouping based on the illusory figures always conflicted with that based on the inducing elements; that is, if the illusory figures biased grouping to the left, the inducing elements biased grouping to the right, or vice versa. In Figure $3 \mathrm{~A}$, for instance, the illusory rectangles should bias grouping toward the left, whereas the orientation of the inducing ovals should bias grouping toward the right.

We did not expect that illusory contours would be the sole influence on grouping performance, because strong similarities among the inducing elements are also present in all displays. To assess whether illusory contours affect grouping, we constructed control displays that contained the same inducing elements, but differently arranged so that they eliminated or weakened the distinctive illusory contours. In Figures 4A and 4B, the positions of the corner inducing elements have been altered to weaken or destroy the perception of illusory figures while leaving all of the inducing elements otherwise intact. Although it is perhaps more difficult to verify in Figures $4 \mathrm{C}$ and $4 \mathrm{D}$ without close scrutiny, these are likewise spa- tially rearranged versions of Figures $3 \mathrm{C}$ and $3 \mathrm{D}$ that weaken the distinctiveness of the illusory contours. The rationale in both cases is that the influence of illusory contours on grouping can be measured by comparing the results of these control stimuli in Figure 4 with the corresponding experimental displays in Figure 3. If grouping is influenced by illusory contours, subjects should be more likely to group the central column with the same illusory figures (i.e., to the left) in Figure 3 than in Figure 4.

The stimuli shown in Figures 5 and 6 were used as filler items to measure the strength of unambiguous grouping in similar stimuli based only on explicit, physically present contours. In the contouronly conditions (Figure 5), the illusory figures of Figure 3 were made explicit and the inducing elements were eliminated. In the elementonly conditions (Figure 6), arrays consisting of just the (completed) inducing elements in Figure 3 were presented. We assumed that in the absence of conflict, grouping would always occur in the unambiguous direction, but we wanted to check this for stimuli similar to those in the experimental and control conditions. These unambiguous stimuli also ensured that subjects would be attending to both global figures and inducing elements on some trials during the experiment.

In each display, square brackets were positioned above and below the central column indicating the column whose grouping subjects were to report. Subjects indicated the direction of grouping (left or right) by pressing the corresponding button on a box that was labeled "left grouping" above the left button and "right grouping" above the right button. They were instructed to press a button as soon as they saw the central column group one way or the other. Subjects were then to indicate the strength of the grouping they reported by pressing a numbered button ( $1=$ weak; $2=$ medium; $3=$ strong). If the subject felt that he/she had accidentally pressed the wrong button on the button box, he/she could press the "error key," which nulled the response and caused the same trial to be retaken later. All stimuli remained on the monitor until a strength rating had been entered.

After subjects had been read the instructions, they were given eight practice trials with stimuli unrelated to the critical stimuli to familiarize them with the procedure and to give them an idea of how the rating scale was to be used. Each subject was recorded for 64 trials consisting of four replications for each condition shown in Figures 3-6.

\section{Results and Discussion}

The results for the baseline conditions (contours only and elements only) were as expected, with $99 \%$ overall grouping in the predicted direction. These data indicate that attention was paid to the task and that subjects reliably grouped with physically present contours for the unambiguous stimuli we used.

The results for the ambiguous experimental and control trials were scored in two ways. First, we calculated the percentage of trials on which subjects reported grouping of the central column with the illusory contours (or its weakened analogue in the control condition) versus the inducing elements. Second, we calculated the average signed rating by scoring the ratings consistent with the illusory figures as positive numbers and those consistent with the inducing elements as negative numbers. Both methods gave essentially the same results.

The percentages of trials on which subjects reported grouping with the illusory contours (or its weakened analogue in the control condition) versus the inducing elements are shown below the experimental and control stimuli in Figures 3 and 4 . The critical comparison is that 

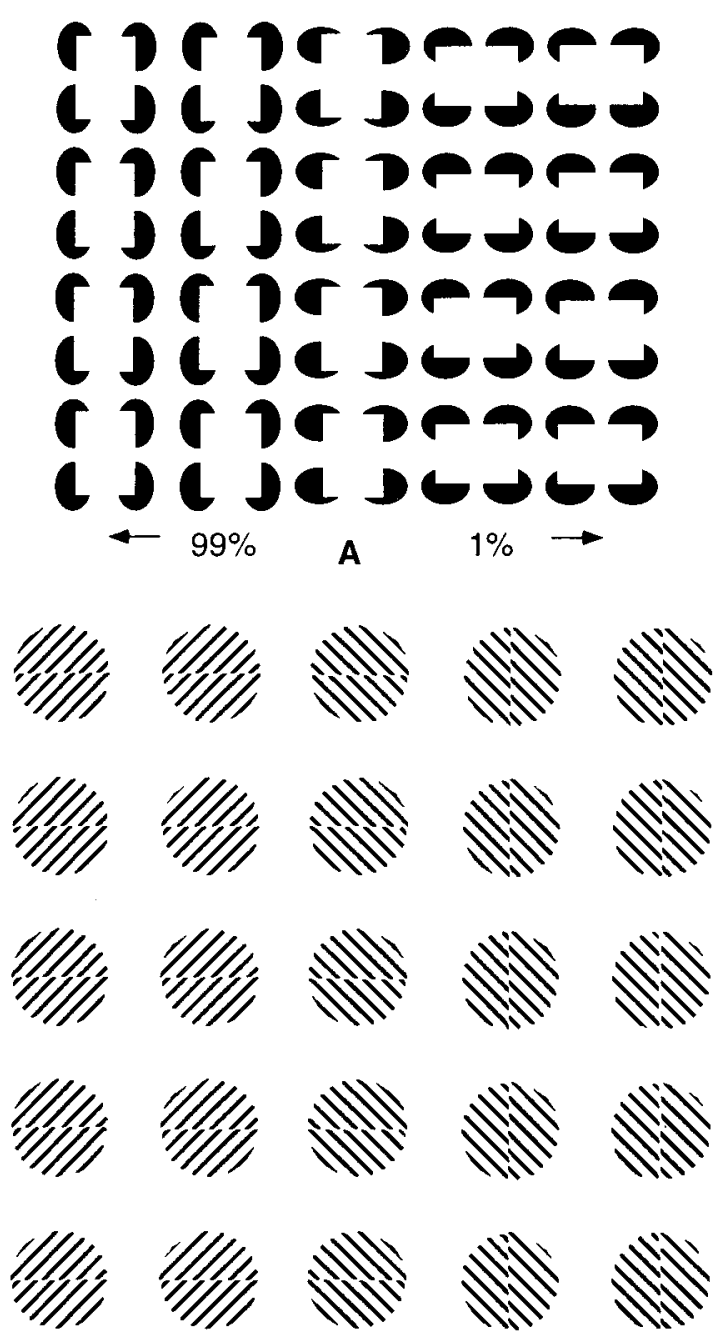

$\leftarrow 81 \%$

$$
\text { C } \quad 19 \% \rightarrow
$$
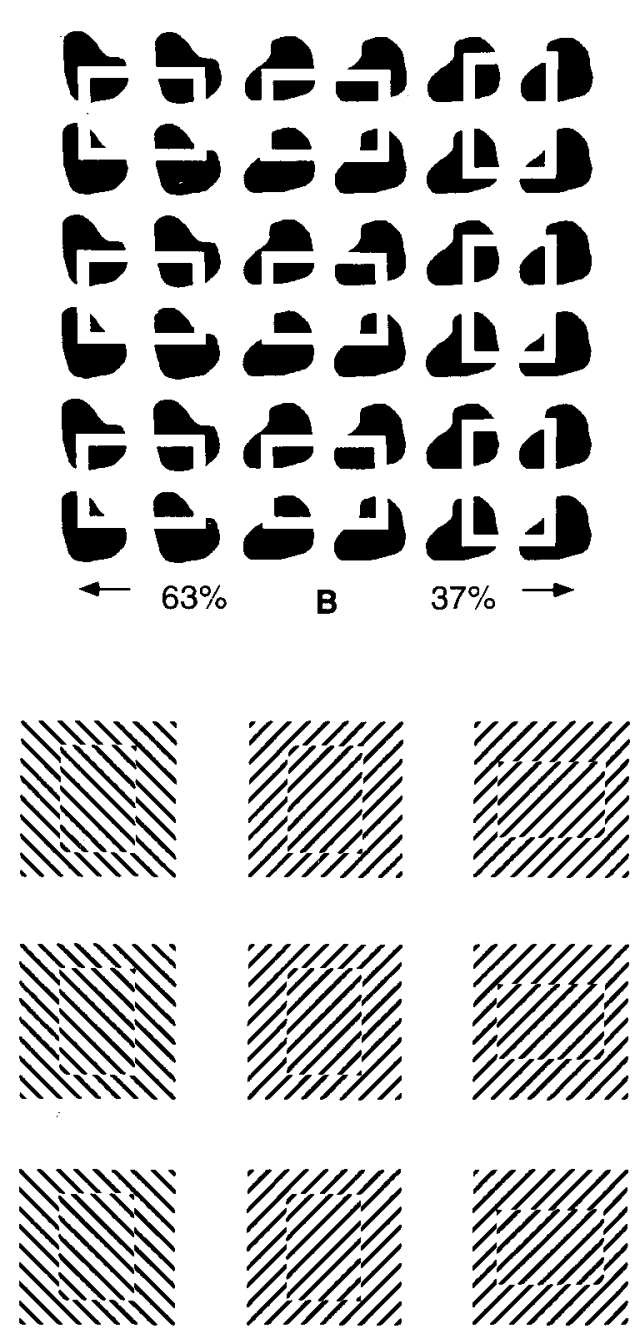

$\leftarrow 73 \%$

D

Figure 3. The ambiguous critical displays used in Experiment 1. A and B show the critical edge-induced illusory figures display, and $C$ and $D$ show the critical line-induced illusory figures display. Numbers below each display to the left and right indicate the percentage of trials on which subjects reported that the central column grouped with the columns on its left and right side, respectively.

between the percentage grouped in the direction predicted by similarity of illusory figures (i.e., to the left in Figure 3 ) versus the corresponding "scrambled" versions in the control conditions (i.e., to the left in Figure 4). In every case, the percentage of grouping responses in the direction biased by the illusory contours was significantly greater than the corresponding percentage in the control condition $[t(27)=9.63,5.16,10.24$, and 13.00, for the rectangles, frames, circles, and squares in Figures 3 and 4, respectively; $p<.001$ in each case]. Indeed, in all four cases, the pattern of grouping reversed for the critical conditions: The majority of responses favored the similar illusory figures in the experimental conditions, but favored the similar inducing elements in the control conditions. These data thus indicate that illusory contours strongly influence perceived grouping. The same pattern was found for the rating data $[t(27)=11.36,5.56,11.70$, and 13.27, for the rectangles, frames, circles, and squares in Figures 3 and 4, respectively; $p<.001$ in each case].

The results thus strongly support the hypothesis that grouping is influenced by illusory figures. Indeed, illusory figures appear to dominate subjective grouping reports with our experimental task and stimuli. The key finding is that removing or weakening the illusory contours of the displays in Figure 3 decisively reversed the pattern of responses. 

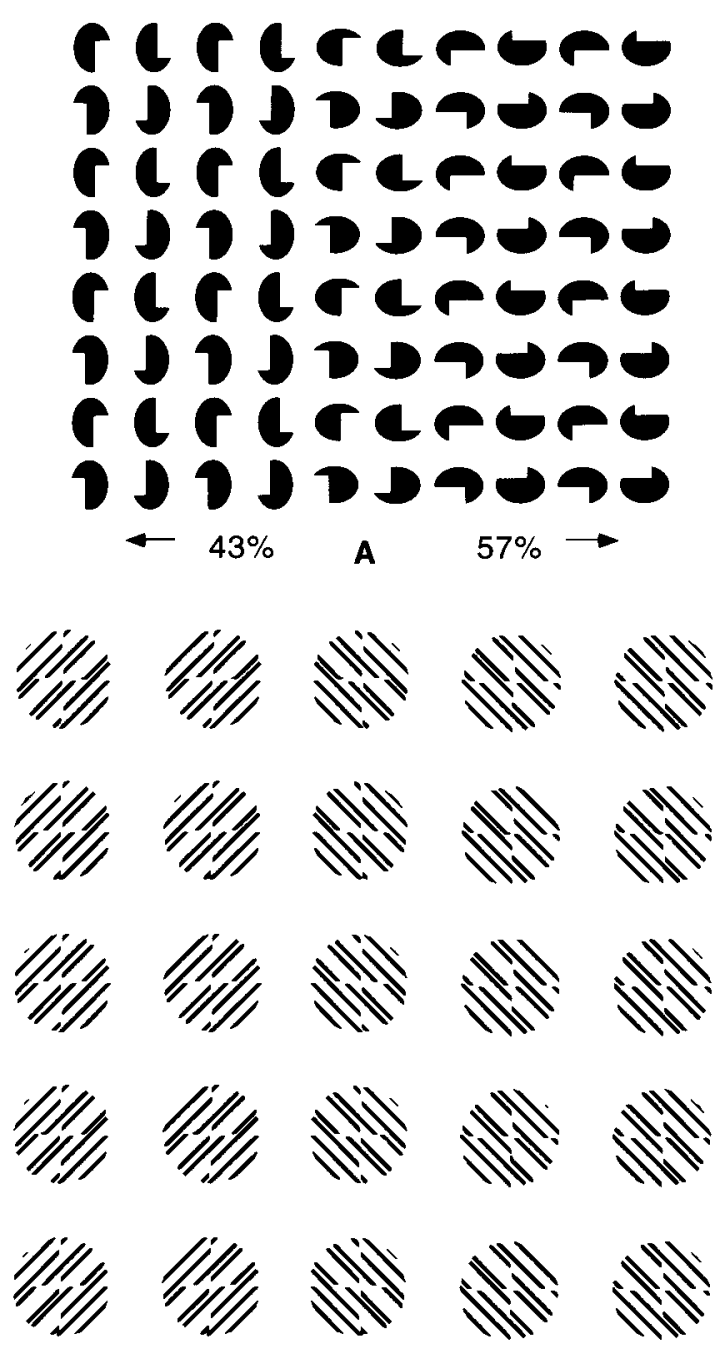

$\leftarrow 8 \%$

$92 \% \rightarrow$
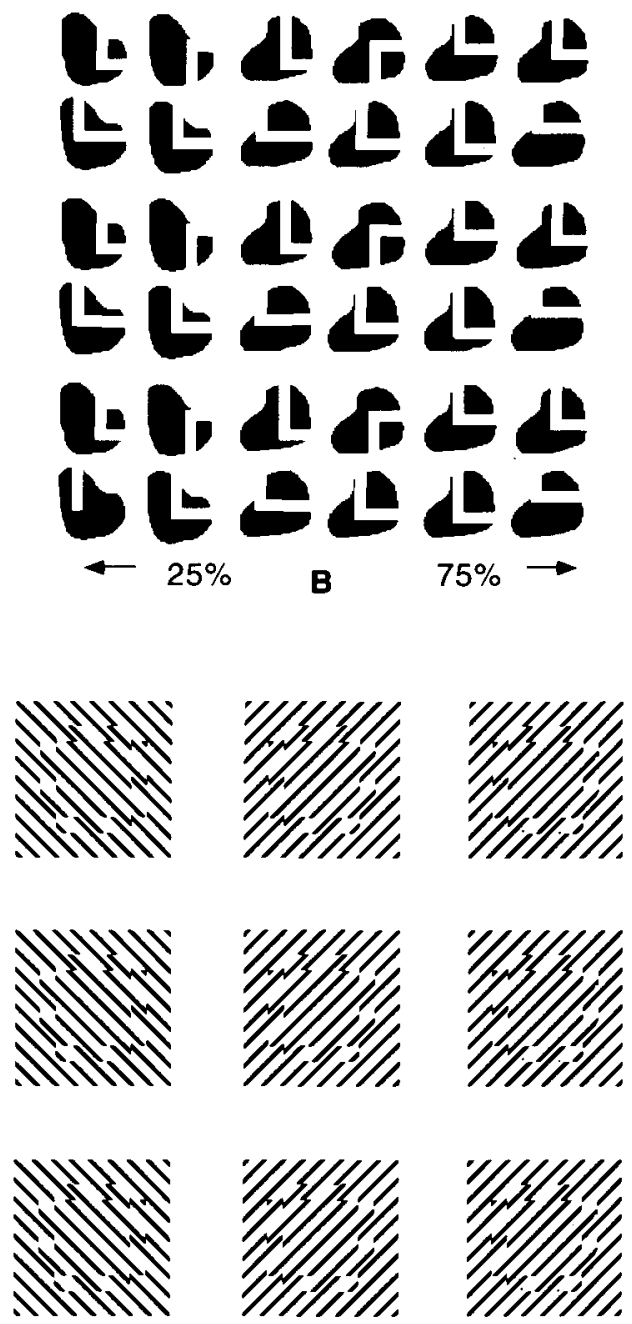

$\leftarrow 4 \%$

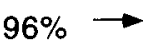

C

Figure 4. The ambiguous control displays used in Experiment 1. The inducing elements in Figure 3 have been rearranged (see text) to weaken or eliminate the perception of illusory figures. Numbers below each display to the left and right indicate the percentage of trials on which subjects reported that the central column grouped with the columns on its left and right side, respectively.

\section{EXPERIMENT 2}

\section{Speeded Performance of Grouping}

Experiment 1 shows that illusory figures can dominate grouping in a subjective report paradigm in which there is no correct answer and subjects are able to view the figures for as long as they want. In Experiment 2, we asked whether this dominance would also be found for objective measures in a speeded performance task.

In Experiment 2, subjects had to perform two different, objectively defined grouping tasks, one based on illusory figures and the other based on inducing elements. In the figure-directed blocks of the experiment, subjects were required to indicate whether the central column grouped with the left or right side of the display specifically with respect to the illusory figures present, independent of the nature of the inducing elements. In the element-directed blocks, they were required to indicate the direction of grouping with respect to the inducing elements, independent of the illusory figures. We measured how quickly and accurately they were able to perform these two tasks. If subjects perform faster when they group according to the illusory figures than when they group according to the inducing elements, then it is reasonable to conclude that the illusory figures dominate perceived organization even under speeded performance conditions. 

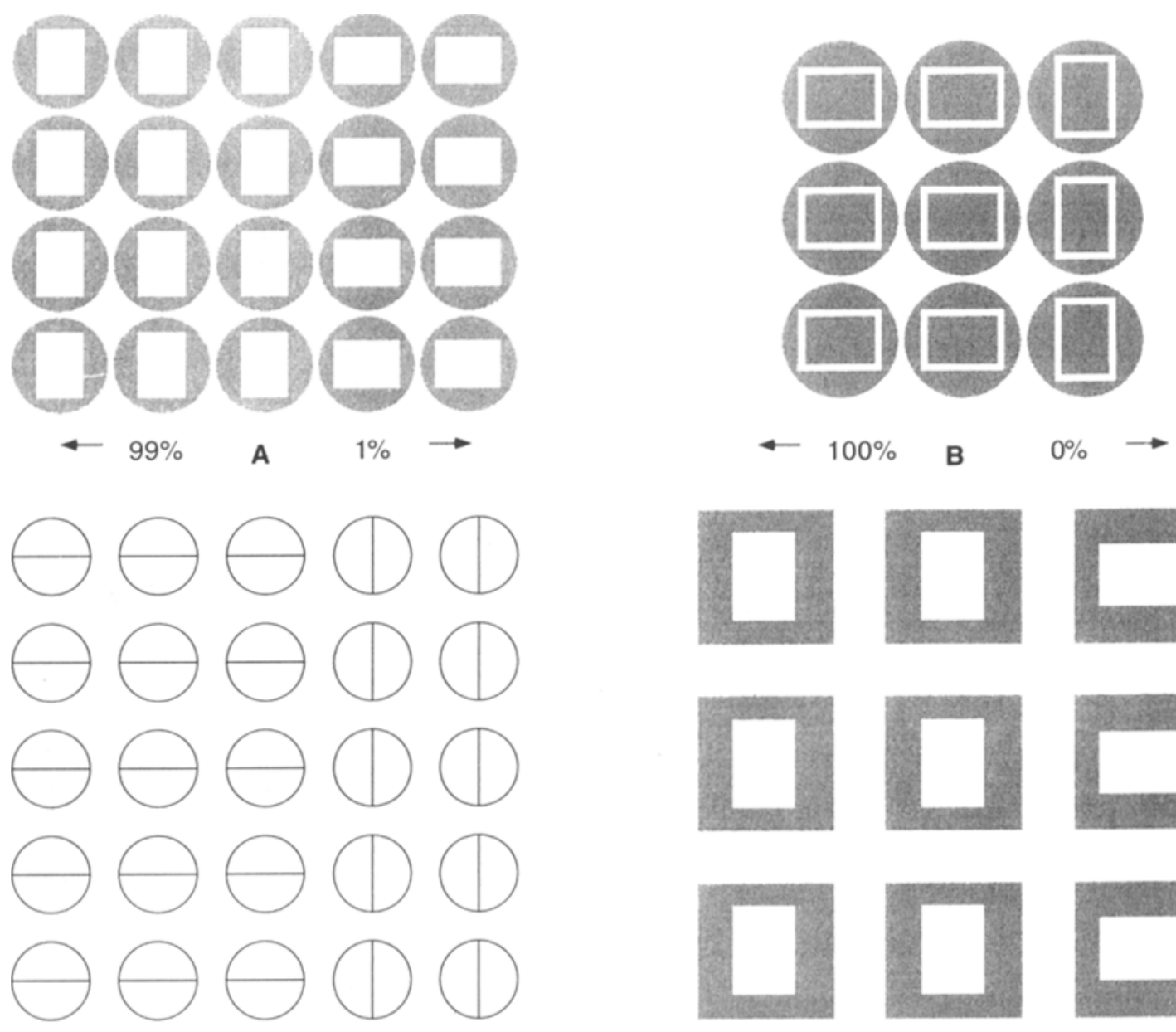

C
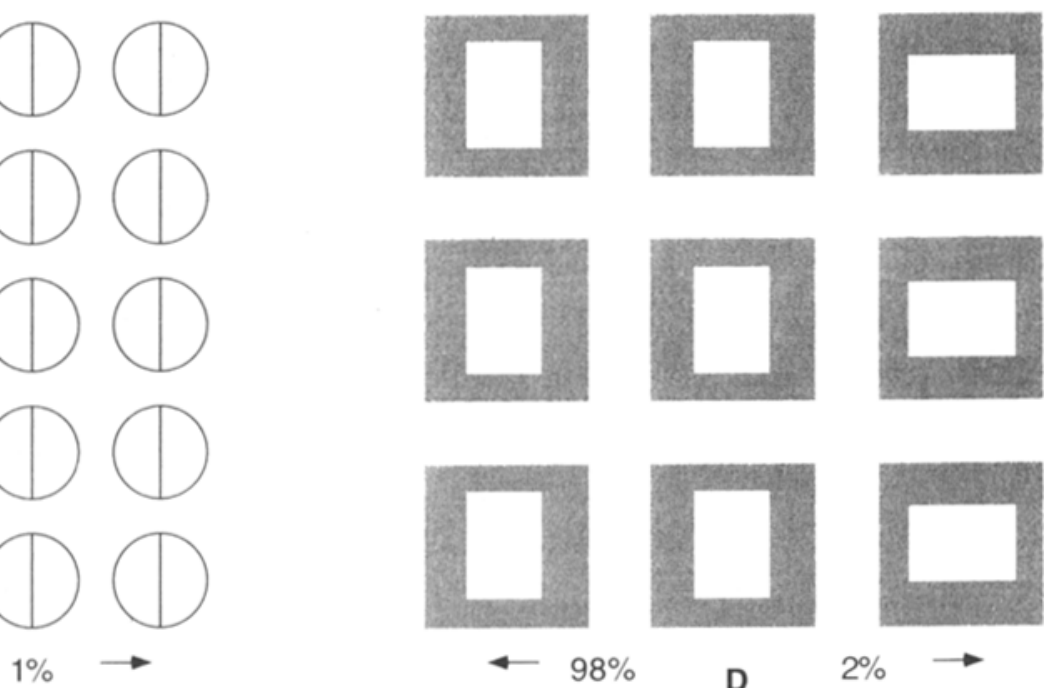

D

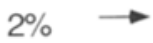

Figure 5. The unambiguous figural displays used in Experiment 1. These displays show just the global figures from Figure 3 without the inducing elements. Numbers below each display to the left and right indicate the percentage of trials on which subjects reported that the central column grouped with the columns on its left and right side, respectively.

As a converging operation, we also studied the effects of interference between illusory figures and inducing elements in this task. In Experiment 1, grouping by illusory figures always conflicted with grouping by inducing elements, but these factors can also be consistent with each other. In Experiment 2, we included both consistent and inconsistent conditions, as shown in Figure 7A and 7B, respectively, for just the rectangle stimuli. In the elementdirected trials, the subjects' task was to indicate the grouping of the central column according to the nature of the inducing elements; in the figure-directed trials, their task was to do so according to the nature of the illusory figure. If illusory figures truly dominate grouping, then consistency should not matter in the figure-directed trials, but consistent conditions should be faster than inconsistent ones in the element-directed trials. We also included corresponding displays in which the figural contours were physically present rather than illusory (Figures $7 \mathrm{C}$ and
7D) to determine how much, if at all, the absence of physical contours matters for performance in these tasks. These contours were added by including a thin black line over the full extent of all illusory contours in every display.

\section{Method}

Subjects. Twenty-five undergraduates from the University of California, Berkeley participated in the study for partial course credit in one of several psychology courses. All were naive with respect to the experimental hypothesis, and all had normal or corrected-to-normal vision.

Materials. The computer and associated equipment were the same as in Experiment 1.

Stimuli and Procedure. Thirty-two stimulus arrays were constructed by the orthogonal combination of the following four factors: the four stimulus types (Figures 3A-3D), the status of the figural contours (illusory or explicit), the side toward which the figure grouped (left or right), and the side toward which the inducing elements grouped (left or right). Each stimulus array was presented in two kinds of trials. In element-directed trials, subjects were instructed to deter- 

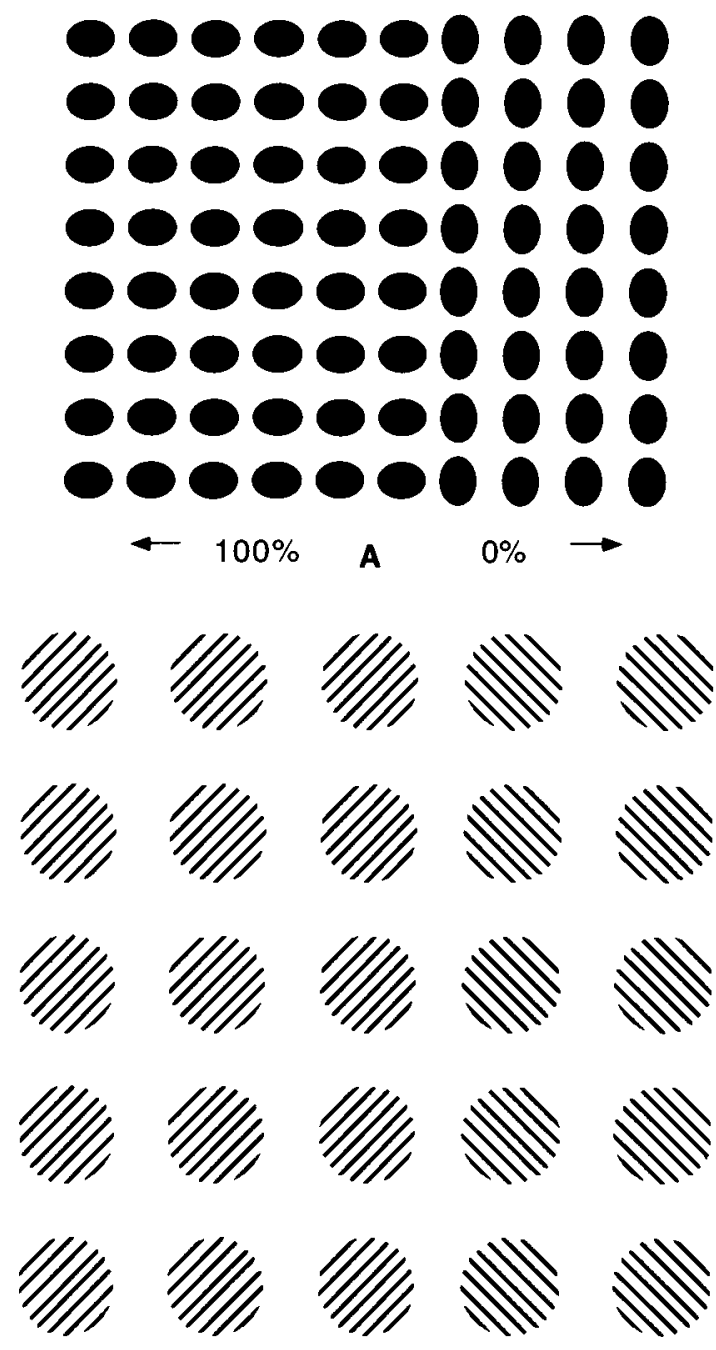

$-98 \%$
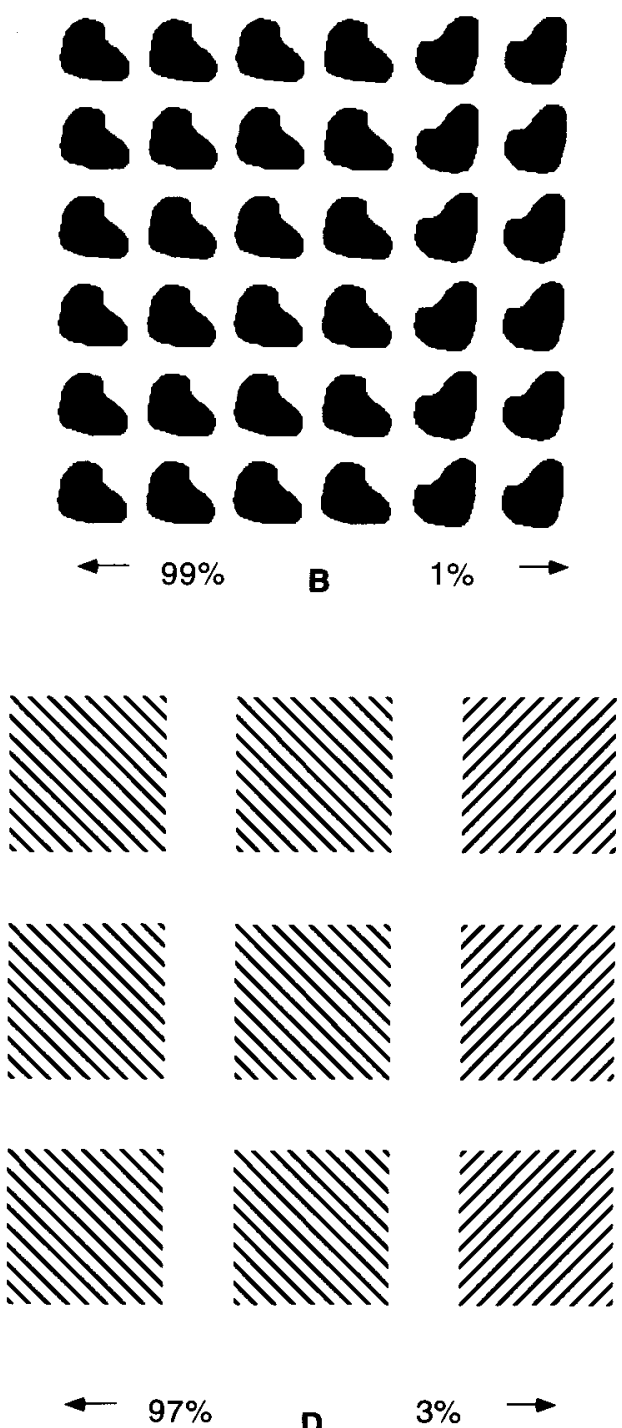

$\mathbf{D}$

$3 \%$

Figure 6. The unambiguous element displays used in Experiment 1 . These displays show just the inducing elements from Figure 3 without any illusory figures. Numbers below each display to the left and right indicate the percentage of trials on which subjects reported that the central column grouped with the columns on its left and right side, respectively.

mine the side toward which the central column grouped according to similarity of the inducing elements. In figure-directed trials, subjects were instructed to determine the side toward which the central column grouped according to similarity of the illusory figures or the corresponding explicit figures in the case of explicit contour stimuli.

The displays were presented in groups of eight blocks, one figuredirected and one element-directed block for each of the four stimulus types. In the figure-directed blocks, the nature of the illusory contour was explained with the aid of an explicit picture. In these figural blocks, subjects were instructed always to group according to the illusory or explicit figural contours and to ignore the inducing elements. In the element-directed blocks, subjects were instructed always to group according to the inducing elements and to ignore the illusory or explicit figures.

Subjects were instructed to make their responses as quickly as possible while keeping their error rate reasonably low. (Feedback was given by sounding a buzzer when subjects made an incorrect response.) Since correct and incorrect responses were well defined for each trial, error trials were discarded and retaken later in the experiment. Only response times on correct trials were analyzed.

Each subject was presented with a total of 320 trials in a six-way factorial design: 5 repetitions $\times 4$ stimulus types (Figures $3 \mathrm{~A}-3 \mathrm{D}$ ) $\times 2$ figural types (illusory or explicit) $\times 2$ relations between figures and inducers (conflicting or consistent) $\times 2$ grouping tasks (elementdirected or figure-directed) $\times 2$ responses (left or right).

\section{Results and Discussion}

The data from 3 subjects were discarded because their error rates were greater than $10 \%$ and/or their response times were more than $3 S D$ above the group mean. The average error rate for the remaining 22 subjects was $3.4 \%$. 
Consistent

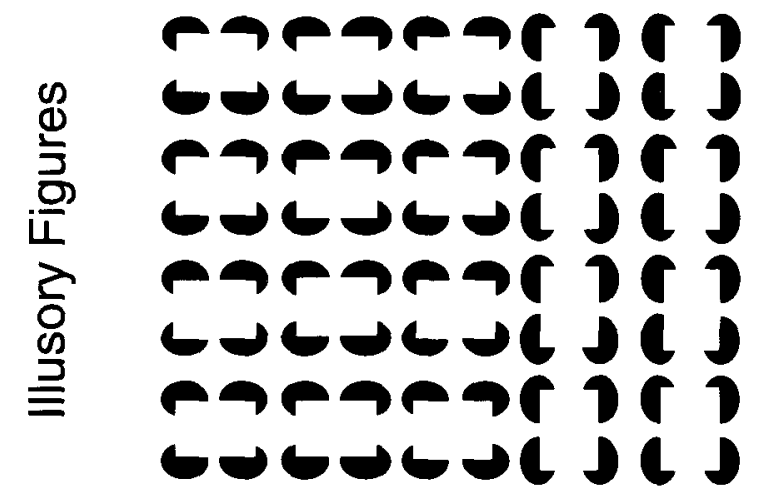

A

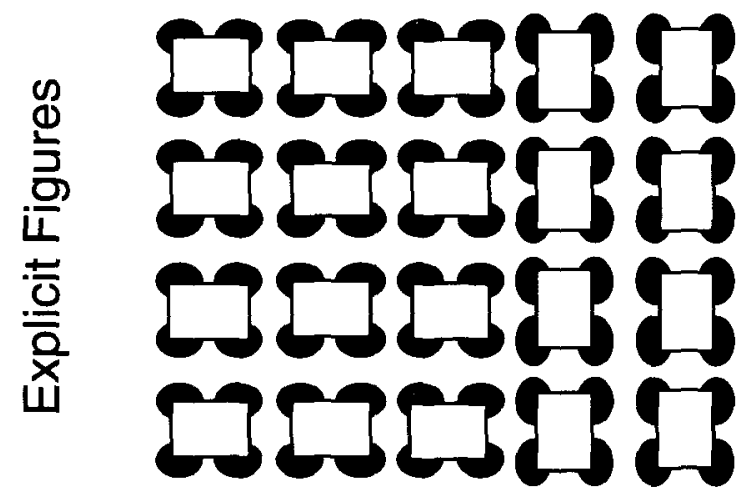

C
Inconsistent

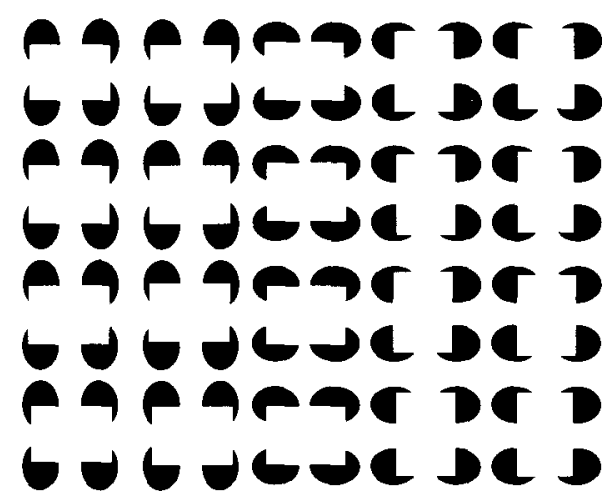

B

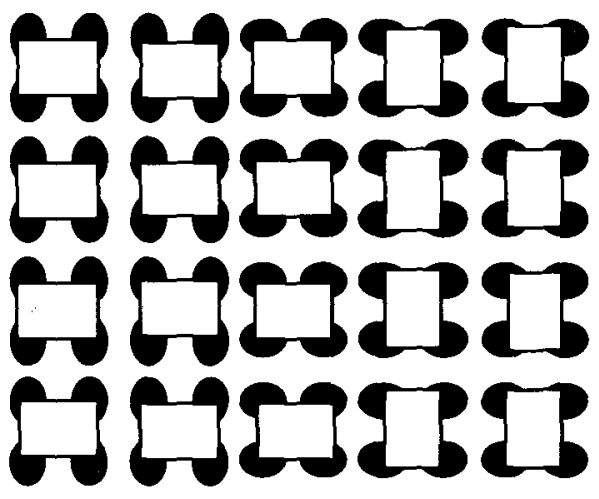

D

Figure 7. Examples of the stimulus displays used in Experiment 2. Global figures were defined either by illusory or explicit contours and defined a grouping structure that was either consistent or inconsistent with the local inducing elements. The example shown corresponds to Figure 3A in Experiment 1, but corresponding versions of Figures 3B, 3C, and $3 D$ were also used.

The average times for correct responses of these subjects were computed and analyzed statistically.

The primary data of interest are plotted in Figure 8, averaged over subjects, repetitions, responses, and stimuli within the edge- and line-induced types. Subjects performed the figure-directed task (circles) faster than the element-directed task (squares) $[t(1,21)=8.26, p<.001]$. This was true for both the edge- and line-induced figures $[t(21)=7.96,6.43, p s<.001$, respectively $]$ and for both explicit figures (solid lines) and illusory figures (dashed lines) $[t(21)=8.62,8.63, p s<.001$, respectively]. These facts are consistent with the hypothesis that grouping by figures was perceptually more salient than grouping by elements, even when the figures were illusory rather than real.
The data also show that subjects responded faster overall to the displays in which the figural and element groupings were consistent than when they were inconsistent $[t(21)=7.05, p<.001]$. However, this difference was much greater when subjects were performing the element task $[t(21)=7.62,5.73, p s<.001$, for edge- and line-induced stimuli, respectively] than when they were performing the figural task $[t(21)=3.15,3.91, p<.01]$. This pattern of results is also consistent with the hypothesis that grouping by figures was perceptually more salient than grouping by elements, because conflicting figural grouping slowed performance in the element grouping task, but conflicting element grouping did not slow performance in the figural grouping task nearly as much $[t(21)=5.38,2.10, p s<.001, .05$, for edge- and 


\section{A. EDGE-INDUCED FIGURES}

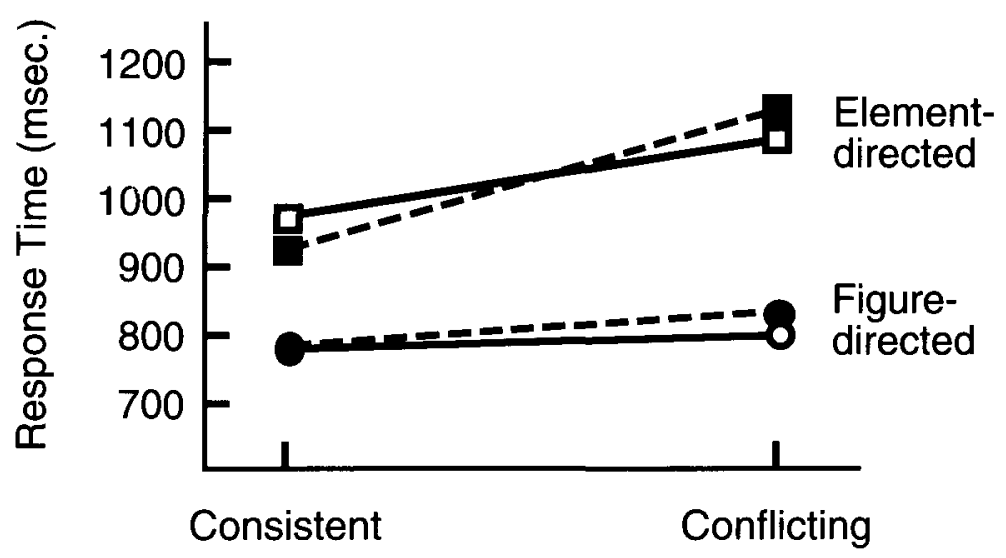

\section{B. LINE-INDUCED FIGURES}

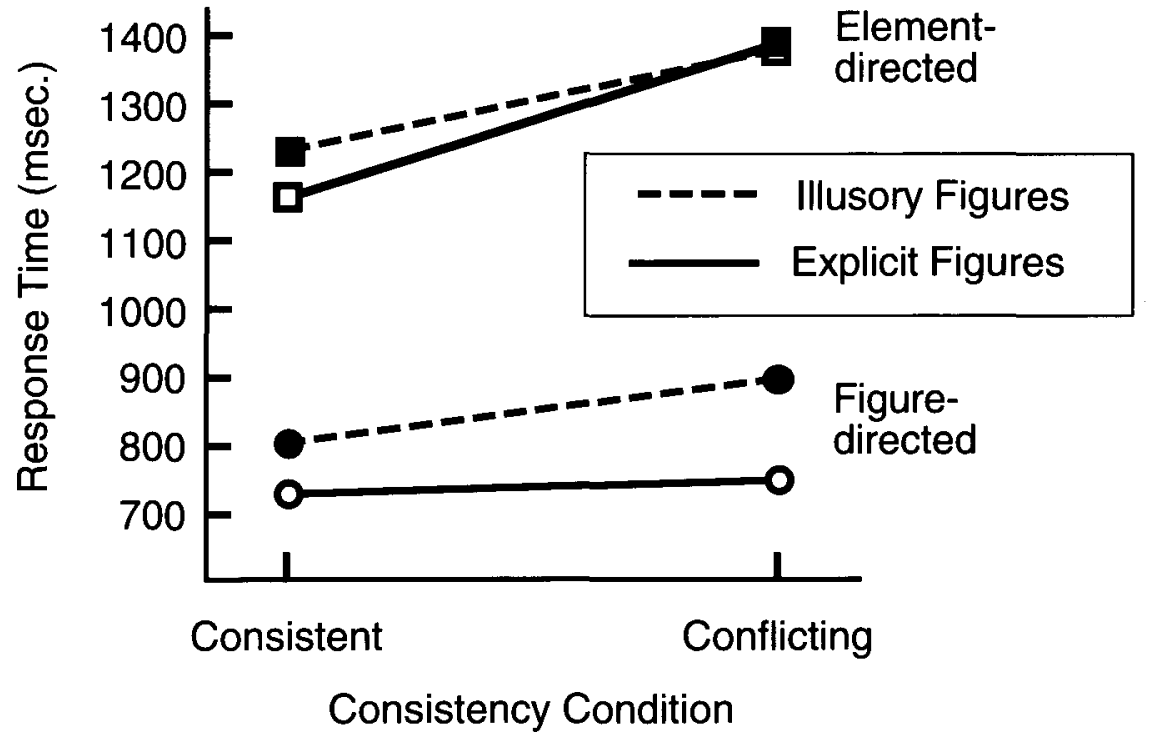

Figure 8. The results of Experiment 2. Response times to report the required grouping of the central column of displays in figure-directed and element-directed tasks are plotted for global figures defined by explicit and illusory contours under conditions in which their grouping is consistent with or conflicts with that based on the inducing elements.

line-induced figures, respectively]. Interestingly, this pattern held regardless of whether the contours of the figures were illusory or explicit, even though explicit contours were responded to overall slightly faster than illusory ones $[t(21)=3.88, p<.01]$.

The qualitative pattern of results described above held for both edge- and line-induced stimulus types, as shown in Figure 8, although the magnitude of the different effects varied somewhat between them. The main difference seems to be that the edge-induced stimuli showed a smaller difference between figure and elements tasks than the line-induced stimuli $\operatorname{did}[t(21)=3.66, p<.01]$. This difference may be due to the relatively lower visibility of the inducing line elements in the line-induced stimuli than the inducing edges in the edge-induced stimuli. It may also be due to the relative discriminability of the differences between the figures and inducing elements, as we will suggest in the General Discussion.

These findings are largely consistent with the hypothesis that grouping by illusory figures dominates grouping by inducing elements in these stimuli. First, grouping based on illusory figures was much faster than that based 


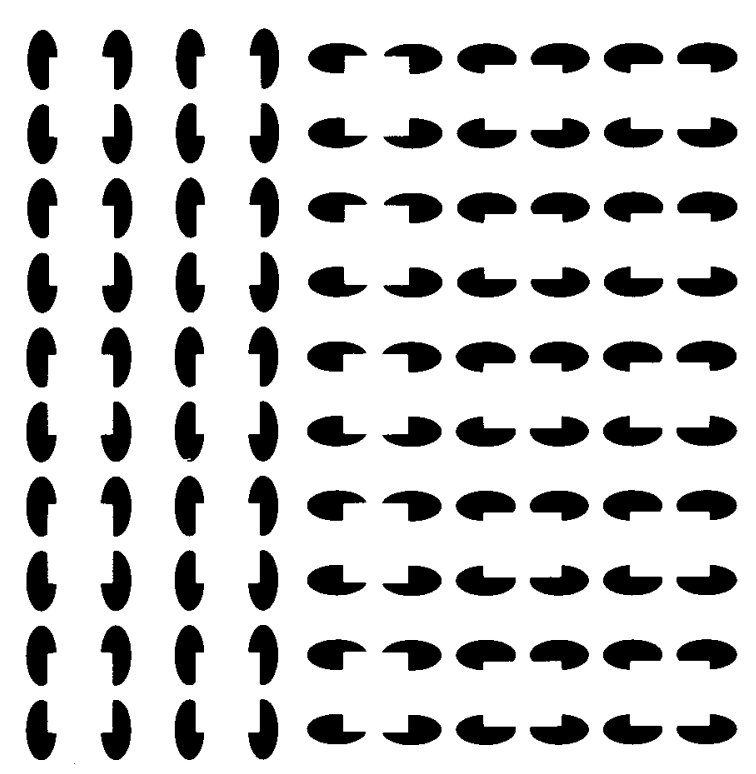

Figure 9. An example in which grouping by inducing elements dominates grouping by illusory figures. This display is qualitatively the same as Figure $3 \mathrm{~A}$ except that the difference in aspect ratios of the inducing elements has been increased, whereas that of the illusory rectangles has been decreased.

on inducing elements for both edge- and line-induced displays. Second, there was more interference from inconsistent figural grouping when subjects were directed to group according to the inducing elements than there was from inconsistent element grouping when subjects were directed to group according to illusory figures. This pattern clearly shows that grouping by illusory figures was stronger than grouping by inducing elements in these stimulus displays. Another result of interest is that, at least for the edge-induced figures, there was virtually no difference in response times between the explicit and the illusory figures. This finding suggests that perceptual grouping is based on a representation that is largely indifferent to whether perceived contours are explicit or illusory, at least for certain types of displays.

\section{GENERAL DISCUSSION}

The results of both experiments support the conclusion that grouping is strongly influenced by illusory contours. Indeed, illusory figures appear to dominate both subjective grouping reports (Experiment 1) and speeded performance measures (Experiment 2), at least with our experimental stimuli and tasks.

We do not claim that this dominance is present in all such displays because it clearly depends on the relative discriminability of the differences between the illusory figures versus those between the inducing elements. Figure 9 shows an example of a display that is qualitatively the same as that in Figure 3A, yet illustrates that inducing elements can sometimes dominate illusory figures in grouping. The reversed dominance results from reduc- ing the difference in the aspect ratios between the two shapes of illusory rectangles in Figure $3 \mathrm{~A}$ and increasing the difference in aspect ratios between the two shapes of inducing elements. This makes the orientational differences between the inducing elements more pronounced than those between the illusory figures. The result is that grouping is now dominated by the inducing elements rather than by the illusory contours.

Does the existence of displays like the one in Figure 9 invalidate the conclusion drawn from the present data that grouping can occur after the perception of illusory contours? We argue that it does not, for at least two reasons. First, we do not claim to have shown that grouping cannot occur before the perception of illusory contours, but only that it can (and does) occur afterward in at least some cases. This conclusion is required by the existence of any displays in which grouping by illusory figures dominates that by inducing elements. Figure 3 shows four such displays, as unequivocally indicated by the results of Experiments 1 and 2.

Second, we contend that the dominance of grouping according to the inducing elements in Figure 9 does not necessarily support the pre-illusory-contours hypothesis that perception of grouping in these cases is based on a representation of the stimulus before illusory contours have been processed. Rather, it is equally compatible with the hypothesis that grouping occurs after illusory contours are perceived. If the "late" representation on which grouping operates includes both the illusory figures and the completed elements that they partly cover, the ambiguity between element-based and illusory-figure-based grouping might arise simply from competition between the conflicting organizations for the illusory figures and the completed elements they cover. No grouping prior to the construction of illusory contours and figures needs to be assumed.

The results of Experiment 2 appear to support the postillusory-figures hypothesis that grouping in all our displays occurs after the perception of illusory contours. The argument goes like this. The alternative to the postillusory-figures account just described is one in which grouping by elements is based on a pre-illusory-figures representation and grouping by figures is based on a postillusory-figures representation. But this hypothesis predicts that grouping by inducing elements should be faster than grouping by illusory figures because the preillusory-contours representation must logically come first. The results of Experiment 2 show that this is not true: Grouping by illusory figures is faster. It therefore seems likely that grouping by both illusory figures and inducing elements occurs after surfaces and occlusion relations have been represented, at least for these displays.

Some readers may object to our characterization of representations that include illusory contours as "late," particularly in light of the findings by von der Heydt and Peterhans (1989; Peterhans \& von der Heydt, 1989) that cells in area $\mathrm{V} 2$ of monkey cortex respond to the presence of illusory contours. Our distinction is logical rather than 
physiological, however, and does not make contact with anatomical location. We claim only that grouping is "late" with respect to the processes that support perception of illusory contours and illusory figures, wherever they may occur in the visual nervous system. If they occur in V2, then at least some processing in V2 is "late" by our definition, even if V2 seems relatively "early" by anatomical criteria. In defense of our view, we note the following two possibilities that bear on the "lateness" of von der Heydt and Peterhans's interpretation of their results: (1) Processing of illusory contours and figures may begin in V2, but may not be completed until some later stage of processing is reached, and (2) cells in V2 may respond to illusory contours in part due to feedback from higher visual areas that include a great deal of further processing. In either case, finding cells responsive to illusory contours in V2 does not indicate that perception of illusory contours corresponds to the initial activation of such cells, and this is the only interpretation under which a good case can be made for an "early" view of illusory contour perception.

The results of the present experiments are thus consistent with the findings of Palmer et al. (1996), Rock et al. (1992), and Rock and Brosgole (1964) that grouping does not operate exclusively on an early, 2-D, imagebased representation, but also on some later representation that includes perceived three-dimensional space, lightness constancy, completed shape, and illusory contours. This conclusion does not warrant the stronger inference that grouping occurs only after these surfacebased representations have been constructed. It is quite possible that grouping occurs throughout visual processing as a bootstrapping process, being continually revised as more sophisticated and veridical representations evolve within the visual system. Image-based representations (e.g., Marr's, 1982, primal sketches) may be organized by some very basic set of principles, such as uniform connectedness (Palmer \& Rock, 1994b) and 2-D versions of grouping factors like proximity, good continuation, similarity, and common fate. These crude organizations may then assist the construction of a representation of surfaces oriented in depth (e.g., Marr \& Nishihara's, 1978, 2.5-D sketch or Barrow \& Tennenbaum's, 1978, intrinsic images) that encorporate the various constancies, depth relations, and figural completions. The result then may require significant revisions in organization, especially when the newly derived intrinsic properties of the surfaces dictate alternative groupings. This new organization can then be used in constructing higher level representations of 3-D volumes and/or object categories, which may in turn produce further reorganizations.

The present results are silent on whether there might be early grouping that occurs before the representation of illusory contours and is then changed once the illusory figures have been perceived. Determining whether this might be so will require a different sort of experiment, one that examines whether grouping factors can be shown to affect the perception of illusory contours. We hope to an- swer this question with further studies using different methods.

\section{REFERENCES}

Barrow, H. G., \& Tennenbaum, J. M. (1978). Recovering intrinsic scene characteristics from images. In A. Hanson \& E. Riseman (Eds.), Computer vision systems (pp. 3-26). New York: Academic Press.

Cohen, J. D., MacWhinney, B., Flatt, M., \& Provost, J. (1993). PsyScope: An interactive graphic system for designing psychology experiments in the psychology laboratory using Macintosh computers. Behavior Research Methods, Instruments, \& Computers, 25, 257-271.

Kahneman, D., \& Henik, A. (1981). Perceptual organization and attention. In M. Kubovy \& J. Pomerantz (Eds.), Perceptual organization (pp. 181-211). Hillsdale, NJ: Erlbaum.

LESHER, G. (1995). Illusory contours: Toward a neurally based perceptual theory. Psychonomic Bulletin \& Review, 2, 279-321.

MARR, D. (1982). Vision: A computational investigation into the human representation and processing of visual information. San Francisco: W. H. Freeman.

MaRR, D., \& NiShihaRA, H. K. (1978). Representation and recognition of the spatial organization of three-dimensional shapes. Proceedings of the Royal Society of London: Series B, 200, 269-294.

NeISSER, U. (1967). Cognitive psychology. New York: AppletonCentury-Crofts.

Palmer, S. E. (1992). Common region: A new principle of perceptual grouping. Cognitive Psychology, 24, 436-447.

PAlmer, S. E. (1999). Vision science: Photons to phenomenology. Cambridge, MA: MIT Press.

Palmer, S. E., \& Levitin, D. (2000). Synchrony: A new principle of perceptual organization. Manuscript in preparation.

Palmer, S. E., NefF, J., \& BeCK, D. (1996). Late influences on perceptual grouping: Amodal completion. Psychonomic Bulletin \& Review, 3, 75-80.

Palmer, S. E., \& Rock, I. (1994a). On the nature and order of organizational processing: A reply to Peterson. Psychonomic Bulletin \& Review, 1, 515-519.

Palmer, S. E., \& Rock, I. (1994b). Rethinking perceptual organization: The role of uniform connectedness. Psychonomic Bulletin \& Review, 1, 29-55.

Peterhans, E., \& von der Heydt, R. (1989). Mechanisms of contour perception in monkey visual cortex: II. Contours bridging gaps. Journal of Neuroscience, $9,1749-1763$.

Rock, I., \& ANSON, R. (1979). Illusory contours as the solution to a problem. Perception, 8, 665-681.

Rock, I., \& BROSGOLE, L. (1964). Grouping based on phenomenal proximity. Journal of Experimental Psychology, 67, 531-538.

Rock, I., Nijhawan, R., Palmer, S. E., \& Tudor, L. (1992). Grouping based on phenomenal similarity of achromatic color. Perception, 21, 779-789.

Spillmann, L., \& Dresp, B. (1995). Phenomena of illusory form: Can we bridge the gap between levels of explanation? Perception, 24, 1333-1364.

Treisman, A. (1986). Properties, parts, and objects. In K. R. Boff, L. Kaufman, \& J. P. Thomas (Ed.), Handbook of perception and human performance (Vol. 2, pp. 35.1-35.70). New York: Wiley.

von Der Heydt, R., \& Peterhans, E. (1989). Mechanisms of contour perception in monkey visual cortex: I. Lines of pattern discontinuity. Journal of Neuroscience, 9, 1731-1748.

WerTheimER, M. (1923). Untersuchungen zur Lehre von der Gestalt: II. Psychologische Forschung, 4, 301-350. [Partial translation in W. D. Ellis (Ed.) (1950). A source book of Gestalt psychology (pp. 71-81). New York: Humanities Press]

(Manuscript received June 16, 1999; revision accepted for publication December 7, 1999.) 